\title{
Maintenance of Intrusive Memories in Posttraumatic Stress Disorder: A Cognitive Approach
}

\author{
Anke Ehlers
}

\section{University of Oxford}

\section{Regina Steil}

\section{University of Göttingen, Germany}

Intrusive recollections are very common immediately after traumatic events and are considered necessary aspects of emotional processing. However, if these intrusive recollections persist over a long time, they are linked to long-term psychiatric disorder, especially Posttraumatic Stress Disorder (PTSD). This paper discusses the need to investigate factors involved in the maintenance of intrusive traumatic recollections. It is suggested that the idiosyncratic meaning of the intrusive recollections predicts the distress caused by them, and the degree to which the individual engages in strategies to control the intrusions. These control strategies maintain the intrusive recollections by preventing a change in the meaning of the trauma and of the traumatic memories. It is further suggested that what is needed is a comprehensive assessment of the processes that prevent change in meaning, going beyond the assessment of avoidance. In particular, safety behaviours, dissociation and numbing, suppression of memories and thoughts about trauma, rumination, activation of other emotions such as anger and guilt and corresponding cognitions, and selective information processing (attentional and memory biases) may be involved in the maintenance of intrusive recollections. Preliminary data supporting these suggestions from studies of individuals involved in road traffic accidents and survivors of child sexual abuse are described.

\section{Introduction}

Even months and years after a traumatic event, patients with Posttraumatic Stress Disorder (PTSD) continue to involuntarily reexperience aspects of the

Acknowledgements: Anke Ehlers is a Wellcome Principal Research Fellow. We are grateful to David M. Clark, Edna B. Foa and Emma Winton for helpful discussions, and to David M. Clark, Paul M. Salkovskis and Emma Winton for helpful comments on an earlier version of this manuscript.

Reprint requests to Dr Anke Ehlers, Department of Psychiatry, University of Oxford, Warneford Hospital, Oxford OX3 7JX, UK.

(C) 1995 British Association for Behavioural and Cognitive Psychotherapies 
trauma. Reexperiencing can take the form of images, thoughts, perceptions, flashbacks, or dreams of the trauma. These intrusive recollections are often considered the hallmark symptoms of PTSD (Calhoun and Resick, 1993; Foa, Steketee and Rothbaum, 1989). They cause significant distress and interfere with the patient's functioning.

The focus of previous work on intrusive recollections, and PTSD symptoms in general, has been on the question of what factors determine whether or not an individual develops psychological symptoms after experiencing a traumatic event. This question is crucial and in many ways the most obvious to study. However, it may not be sufficient in explaining PTSD. The aim of the present article is to argue that for a full understanding of the nature of PTSD, research will have to clarify which factors determine the maintenance of PTSD symptoms. Research on rape and assault victims has demonstrated that the majority of victims meet the criteria for PTSD in the weeks following the trauma (Rothbaum, Foa, Riggs, Murdock and Walsh, 1992; for reviews see Kilpatrick and Resick, 1993; Rothbaum and Foa, 1993). While the majority recover in the course of the following year, a proportion show persistent symptoms. Research on other types of trauma has often found a lower prevalence of PTSD, but nevertheless symptoms of PTSD such as intrusive recollections were very common immediately after the trauma (e.g., Shalev, 1992; Shalev, Schreiber and Galai, 1993). Thus, it seems to be important to determine which factors explain maintenance versus recovery in individuals initially showing tite characteristic posttraumatic symptoms as specified in the DSM-IV diagnoses Acute Stress Disorder or PTSD (American Psychiatric Association, 1994) after a traumatic event.

The need to clarify which factors determine maintenance particularly applies to intrusive memories of the trauma since most researchers agree that for the period immediately following the trauma, reexperiencing is a sign of normal adaptation. For example, Eberly, Harkness and Engdahl (1991) pointed out that in a traumatic environment "intrusive replaying of trauma in dreams and while awake allows the organism to rehearse covertly alternative responses and contemplate the overall unpleasantness of the situation so that avoidance or escape from the life-threatening situation becomes more likely if encountered again" (p. 367). Persistent intrusive memories, on the other hand, are linked to poor long-term outcome and may be involved in maintaining the disorder: "... intrusive memories may be a primary mechanism by which the experience of stress was extended beyond the presence of the stressor" (Baum, Cohen and Hall, 1993, p. 282).

This paper is concerned with possible cognitive factors involved in the maintenance of intrusive memories in PTSD. It presents a brief overview 
of cognitive factors established in previous research as well as suggestions for possible further cognitive processes that may maintain intrusive memories. These suggestions are supported by preliminary findings from our studies on individuals involved in road traffic accidents and survivors of child sexual abuse.

It is not the aim of this paper to present a complete overview of possible variables contributing to the development of PTSD (e.g., variables such as prior psychiatric history or prior life events; see Jones and Barlow, 1990, for a review). It is also outside the scope of this paper to review possible biological factors involved in PTSD symptoms. We do not see biological and cognitive factors as mutually exclusive and assume that cognitive processes have biological correlates (see, for example, the relationship of numbing and endogenous opiates, Glover, 1992; van der Kolk and Saporta, 1991; relationships of hyperarousal, stress hormones, and disturbances in memory, van der Kolk and Saporta, 1991; or a possible relationship between stress hormones and overconsolidation of memories, Baum et al., 1993).

\section{Cognitive factors involved in the development of PTSD symptoms}

Individuals exposed to the same trauma differ widely in the degree of their distress. This points to the role of cognitive factors in that it seems to be important how an individual perceives the trauma and what meaning the trauma has for him or her. Psychological models and empirical studies have addressed cognitive factors that might explain the range of reactions to trauma. Since these provide the background for the discussion of maintenance, a few important factors are now described, with some examples of research findings supporting the role of these factors.

\section{Unpredictability and uncontrollability}

Most authors agree that the experience of unpredictability and uncontrollability represent a core cognitive factor for the development of PTSD (Davidson and Foa, 1993; Foa, Zinbarg and Rothbaum, 1992; Jones and Barlow, 1990; Rachman, 1990). "For the victim of an uncontrollable, unpredictable important stressor, daily life is marked by a sense of impending doom because threat pertinent to his or her survival can neither be predicted nor controlled" (Foa et al., 1992, p. 231). Foa et al. $(1989,1992)$ specified that the concept should be understood as perceived controllability and predictability rather than actual contingencies and that controllability may even play a more pivotal role that predictability in determining PTSD outcomes. In line with this hypothesis, prospective data from individuals living near the Three Mile Island nuclear accident showed that perceived loss of control was a significant mediator of stress response (Baum et al., 
1993). For a review of further studies supporting the role of unpredictability and uncontrollability of the trauma, the reader is referred to Jones and Barlow (1990) and Foa et al. (1992). Further aspects of control are discussed elsewhere; for example, the perception of unexercised control (Foa et al., 1989) and the role of disruption of expectations of control (Baum et al., 1993).

\section{Perceived threat vs. actual danger}

Research indicates that the perception of threat is at least as important in predicting the severity of PTSD symptoms as objective indicators of actual danger (Foa et al., 1992). For example, Kilpatrick et al. (1989) found that rape victims who perceived the assault to be life-threatening were more likely to develop PTSD than those who did not think their life was in danger. In contrast, whether or not a weapon was used in the crime was not related to PTSD. Furthermore, multiple regression analysis showed that perceived threat to life explained a proportion of the variance of development of PTSD not explained by physical injuries sustained during the assault. Using path analysis, Riggs, Foa, Rothbaum and Murdock (1991) found that for rape and crime victims, the relationship between severity of assault and severity of PTSD was mediated by perceived threat to the victim's life. In a questionnaire study of 375 subjects involved in road traffic accidents, the perceived threat to life was also related to severity of PTSD symptoms (Steil and Ehlers, in preparation). The same result was obtained in a three-month prospective study of road traffic accident victims (Winter and Ehlers, in preparation).

An aspect of perceived threat that has not been adequately addressed in the existing literature is the fact that an individual's memory of how threatening the event was may change over time (see for example Schwarz, Kowalksi and McNally, 1993) and new information may be incorporated into the memories of perceived threat. Foa et al. (1989) described the case of a rape patient who only developed PTSD after hearing that the perpetrator had killed his next victim. In a questionnaire survey of 159 individuals involved in road traffic accidents, subjects meeting the criteria for PTSD did not differ in their ratings of how threatening they perceived the accident while it happened from subjects without PTSD. However, significant differences were found when subjects rated how threatening the accident appeared from today's perspective (Steil and Ehlers, in preparation). Parallels exist between these observations and the phenomenon of UCS revaluation in conditioning (Davey, 1989). 


\section{Causal attributions}

There is some evidence that causal attributions are related to the development of PTSD (see Joseph, Yule and Williams, 1993 for a review). Subjects with PTSD tend to attribute the trauma to more internal causes than traumatized individuals without PTSD. There is also evidence that general attributional style is correlated with PTSD in that individuals with PTSD show more externality for positive outcomes. Evidence for negative outcomes is mixed and might depend on the type of trauma (Joseph et al., 1993). Wenninger and Ehlers (submitted) found that highly symptomatic survivors of child sexual abuse attributed negative events to more internal, stable, and global causes than mildly symptomatic survivors. In a prospective study of individuals involved in road traffic accidents, subjects who met criteria for PTSD were more likely to attribute negative events to internal causes than subjects who did not develop PTSD (Winter and Ehlers, in preparation).

\section{Effects of trauma on beliefs}

Janoff-Bulman and Frieze (1983) hypothesized that psychological symptoms develop after a trauma if an individual's basic assumptions about the fairness and safety of the world are shattered. Other authors have also emphasized the perception of loss of safety/increased risk in PTSD patients (e.g., Foa et al., 1989). Foa and Riggs (1993) and Resick and Schicke (1993) pointed out that the trauma may not only shatter previous beliefs but may also confirm preexisting maladaptive beliefs. Individuals with exaggerated notions about the safety of the world and about their personal invulnerability, and individuals with exaggerated ideas of danger and personal incompetence may be more likely to develop PTSD than individuals with more flexible schemata (Foa and Riggs, 1993). In general, the trauma may have different implications on the individual's view of themselves, their relationships with other people, and their expectations for their future. For example, persistent PTSD in rape victims is thought to be due to maladaptive cognitions the victim develops because the assault conflicted with prior schemata in themes such as safety, self-esteem, competence, trust and intimacy (Calhoun and Resick, 1993; McCann and Perlman, 1990). In line with this assumption, Wenninger and Ehlers (submitted) found high correlations between dysfunctional beliefs in these themes and trauma-related symptoms in adult survivors of child sexual abuse. These correlations were not mediated by severity of assault. 


\section{Coping strategies}

In their review of the literature, Jones and Barlow (1990) pointed out that coping strategies may be an important variable moderating an individual's response to a trauma. Active problem-focused processing strategies seem to be linked to better outcome than avoidance or emotion-focused strategies such as distancing or wishful thinking (see for example, Fairbank, Hansen and Fitterling, 1991; Solomon, Mikulincer and Avitzur, 1988; Solomon, Mikulincer and Flum, 1988). In line with this hypothesis, a coping style characterized by the factor escape/avoidance as measured by the Ways of Coping Scale predicted severity of PTSD symptoms three months after road traffic accidents (Winter and Ehlers, in preparation).

\section{Cognitive mediation of other variables}

Some other variables that have consistently been found to be linked to outcome in individuals who are exposed to traumatic events may also be mediated by cognitive processes. An example is social support, which is linked to good outcome (see Flannery, 1990; Jones and Barlow, 1990). Little is known about the mechanisms by which social support prevents the development or maintenance of PTSD symptoms and one can only speculate about possibilities. For example, social support may give the individual a sense of controllability (Jones and Barlow, 1990), confiding in others may be therapeutic (Pennebaker, 1989), and social support may prevent maladaptive interpretations of trauma (such as "I will not be able to have close relationships again", "I am worthless"). Feeling detached from others may represent a form of dissociation which prevents adjustment (see suggestion 2 below), and loss of social support may represent a long-lasting loss of resources (Freedy, Shaw, Jarrell and Masters, 1992).

\section{Conclusions}

Previous research has established a number of cognitive factors that are involved in the development or maintenance of PTSD symptoms after traumatic events. The focus of cognitive theories and research has been on the individual's perception of the trauma. Two research strategies may increase the explanatory power of cognitive factors in PTSD. First, a clearer distinction of factors linked to the first development of PTSD symptoms and those determining their persistence may be useful. Because most studies to date are retrospective, there is a lack of data relevant to this distinction. Second, cognitive factors involved in the maintenance of PTSD may go beyond those related to the trauma itself and may include the patients' 
interpretations of their PTSD symptoms, as in panic disorder (Clark, 1986; Ehlers, Margraf and Roth, 1988).

\section{Research on intrusive recollections in PTSD}

\section{Descriptive information on the occurrence of intrusive memories}

Some authors have found that avoidance symptoms are more persistent than symptoms of the reexperiencing cluster (for a review see Blank, 1992). However, a closer inspection of the data shows that differences exist between the different symptoms of the reliving cluster. A study of survivors of the Buffalo Creek dam collapse (cited from Green, 1993) found that while nightmares and flashback were relatively uncommon 14 years after the disaster, intrusive thoughts and psychological and physiological distress when confronted with reminders of the trauma were among the most common long-term symptoms of PTSD. In a three-month prospective longitudinal study of 39 individuals involved in road traffic accidents, Winter and Ehlers (in preparation) obtained similar results. Three months after the accident, $48 \%$ of the subjects still experienced intrusive recollections and $54 \%$ experienced distress when confronted with reminders of the trauma, whereas only $10 \%$ experienced nightmares and $26 \%$ flashbacks. Cognitive avoidance was reported by $31 \%$ of the subjects, and behavioural avoidance by $39 \%$.

It is important here to note that the label "intrusive thoughts" that has been used by some authors to represent criterion B1 of DSM-III-R and DSM-IV (American Psychiatric Association, 1994) is misleading because intrusive recollections include images of the traumatic event. Brett and Ostroff (1985) remarked that the role of imagery is underinvestigated in PTSD. To date, there are insufficient data about the nature of intrusive recollections. According to our results, visual recollections are the most common form of intrusions. In a study of 35 adult survivors of child sexual abuse, Wenninger and Ehlers (in preparation) found that $97 \%$ reported visual recollections $(51 \%$ in the resembling "film clips", $46 \%$ resembling single pictures), $66 \%$ somatosensory sensations, $51 \%$ sounds or smells, and $26 \%$ thoughts.

Steil and Ehlers (in preparation) obtained similar results in a sample of 159 individuals who had experienced road traffic accidents. $65 \%$ of the subjects reported visual recollections resembling "film clips", $69 \%$ seeing single pictures, $54 \%$ sounds or smells, $48 \%$ somatosensory sensations, $45 \%$ thoughts, and $33 \%$ felt as if they were repeating actions performed during the accident. Furthermore, Steil and Ehlers (in preparation) obtained distress ratings for each of the symptoms of PTSD and found that the symptoms 
of the reliving cluster were rated as more distressing than symptoms of the avoidance/numbing and arousal clusters. Visual recollections were rated as more distressing than other recollections.

Taken together, these results suggest that models explaining maintenance of traumatic memories need to take their visual quality into account. The predominance of intrusive visual images might be an important difference between PTSD and other anxiety disorders with frequent intrusions such as Generalized Anxiety Disorder (GAD).

\section{Maintenance of intrusive memories in models of PTSD}

There is general agreement that reexperiencing is necessary for emotional processing (see Rachman, 1990) and is thus to be expected in all traumatized individuals for a certain period after a trauma. From differing theoretical perspectives, Horowitz (1976) and Foa et al. (1989) have both suggested that intrusive memories persist if emotional processing is prevented or incomplete due to avoidance strategies (see also Chemtob, Roiblat, Hamada, Carlson and Twentyman, 1988; Janov-Bulman, 1985).

According to Horowitz (1976), intrusive memories facilitate information processing of the trauma by two processes, first, incorporation of the trauma into existing schemata, and second, by the development of new schemata that take the trauma into account. Avoidance and numbing are seen as control processes that prevent the individual from becoming overwhelmed by the memories. Horowitz describes the adaptation process as an oscillation between denial and numbing on the one hand and intrusive memories on the other hand. Excessive control of the intrusive memories, however, will prevent complete processing of the trauma and thus prolong the adaptation process. The trauma will stay in active memory and intrusions will continue to occur.

On the basis of Lang's (1977) network model of emotion, Foa et al. (1989) suggested that traumatic events create especially large and complex fear networks because of their significance and because they violate formerly held basic concepts of safety. Because of their complexity, fear networks of PTSD patients can easily be activated by a wide range of stimuli. This leads to a sense of unpredictability and uncontrollability and is involved in maintaining the disorder. According to Foa et al. (1989) the persistence of PTSD can be explained by inadequate activation of the fear network due to several mechanisms: avoidance strategies and numbing prevent an adequately long activation of the fear network, the complex network may not be activated entirely because not enough matching cues are present or because memories of the traumatic event are fragmented and disorganized 
(Foa and Riggs, 1993), and/or excessively high arousal impedes habituation and processing of new information.

\section{Relationship of intrusive memories, avoidance, and psychiatric disorder}

Models of PTSD differ in the relationship proposed between intrusion and avoidance. Horowitz (1976) and Chemtob et al. (1988) distinguish phases of intrusive memories accompanied by high arousal and phases of arousalreducing avoidance as separate modes of functioning. The patient is thought to oscillate between these modes. Chemtob et al. (1988) assume that the mode characterized by hypervigilance for threat, intrusions, and high arousal and the mode characterized by avoidance of threat and reduction of arousal represent two separate network nodes in memory which mutually inhibit each other. Foa et al. (1989) and Creamer, Burgess and Pattison (1992) see avoidance as a direct part of the fear structure associated with the trauma (the fear structure is a program to escape or avoid danger) and emphasize that intrusions motivate the patient to avoid reminders of the trauma.

Recent research supports the view of a functional relationship between intrusions and avoidance. McFarlane (1992a) studied firefighters exposed to a bushfire over a period of $\mathbf{4 2}$ months. Using path analysis, he demonstrated that intrusive recollections predicted degree of avoidance at all time points $(4,11$, and 29 months, $N=395)$. Creamer, Burgess and Pattison (1990) analysed two data sets (one of an earlier study of McFarlane) with path analyses and found that, consistent with McFarlane's (1992a) data, intrusions precede avoidance. Similarly, in a 14-month prospective study of 158 individuals exposed to a multiple shooting in their office building (Creamer et al., 1992) intrusions predicted avoidance, but not vice versa, at all time points $(4,8$, and 14 months).

At first sight, the path analysis models presented by Creamer et al. (1992) and MacFarlane (1992a) on the role of intrusive recollections in predicting long-term psychiatric symptoms after exposure to a trauma seem to differ. McFarlane (1992a) found that intrusive recollections measured at 4 months predicted psychiatric disorder (PTSD, other anxiety disorder, or depression, according to DSM-III criteria) at 42 months $(N=147)$. Interestingly, avoidance did not have an independent relationship to the disorder. Like MacFarlane (1992a), Creamer et al. (1992) found positive correlations between intrusions at earlier phases and subsequent SCL-90R scores (which were used in this study as an indicator of psychiatric disorder-intrusions at 4 month with SCL-90R at 8 months: $r=.60$, with SCL-90R at 14 months: $r=.54)$. LISREL modelling showed that intrusive recollections resulted in higher SCL-90R scores, but not vice versa, at all time points. Intrusions at 
earlier stages showed strong positive relationships with subsequent intrusions (which were linked with higher SCL-90R scores at that time point), and small but significantly negative relationships with subsequent SCL-90R scores. The authors interpret the latter finding as meaning that intrusions at earlier stages are a sign of cognitive processing and are therefore related to reduced symptoms in the long-term. However, one has to bear in mind that intrusions at earlier stages and SCL-90R scores at later stages correlated positively (as in McFarlane's, 1992a, study). The pattern of results seems to indicate that a proportion of the variance of intrusive recollections at earlier phases is not predictive of subsequent intrusive memories, and it is that part of the variance that shows a negative relationship to subsequent psychiatric disorder. However, most of the variance of intrusive recollections at 4 months seems to predict poor long-term outcome, as in MacFarlane's model. A consistent finding of both studies is that intrusive recollections, but not avoidance, predicted psychiatric impairment about one year after the trauma.

Another interesting finding of the Creamer et al. (1992) study is that the link between intrusive memories and avoidance is close a few months after the event but that the size of the correlation decreases over time (although MacFarlane, 1992a, found similarly high regression coefficients for all time points). Other authors have also pointed out that correlations between avoidance and intrusive memories is often low, especially if measured a long time after the trauma. This has led authors to conclude that other factors than intrusions contribute to avoidance behaviour (e.g., Burstein, 1989a). For example, MacFarlane (1992a, 1992b) suggested that avoidance presents a variable reaction to the intrusive recollections and may partially reflect the individual's personality such as neuroticism or genetic differences. Using cross-lagged panel correlations, MacFarlane (1992a) found a positive feedback process between intrusive recollections and symptoms of anxiety and depression. As we will discuss below, cognitive variables that may mediate an individual's response to having intrusive recollections should also be taken into account (see suggestion 1). Furthermore, the inclusion of more subtle forms of avoidance may be important (see suggestion 2).

\section{Relationship of intrusive memories and distress}

Most of the earlier work on PTSD implicitly assumed that intrusive memories are direct expressions of trauma-induced distress (see for example Creamer et al., 1990; Feinstein and Dolan, 1991; MacFarlane, 1992a), and the intrusions scale of the Impact of Event Scale (IES, Horowitz, Wilner and Alvarez, 1979) which assesses how often intrusive symptoms are experi- 
enced has been widely used to assess psychological distress induced by the trauma.

Preliminary studies, however, indicate that individuals vary in the degree to which they find intrusive recollections distressing. Shalev et al. (1993) observed that during the week after the trauma, all survivors of a terrorist attack experienced intrusive thoughts and images, but that "substantial differences between individual's capacity to tolerate the intrusive phenomena were present. These were expressed by the amount of distress and arousal associated with the emergence of traumatic recollection" (p. 446). Similar findings from our research of individuals involved in road traffic accidents are described below (Steil and Ehlers, in preparation). Mayou, Bryant and Duthie (1993) found that if patients described their intrusive recollections of road traffic accidents as "horrific" (which can be taken as a sign of distress) they were likely to meet criteria for PTSD at 1-year follow-up. Although it is difficult to equate for the exact content of the intrusive recollections in these studies, they point to the important distinction between frequency of intrusions and distress caused by them. The latest revision of the Diagnostic and Statistical Manual of Mental Disorders (DSMIV, American Psychiatric Association, 1994) has added a distress criterion to take into account that the occurrence of symptoms does not necessarily reflect intensity of distress.

The failure to distinguish between frequency of intrusive recollections and the distress caused by them in most previous research on PTSD is somewhat surprising, given that Rachman's group (e.g., Parkinson and Rachman, 1981; Rachman and de Silva, 1978) has demonstrated that the occurrence of unwanted thoughts does not necessarily mean that they are distressing and that frequency of unwanted intrusive thoughts represents a different factor from discomfort and controllability factors. If applied to PTSD, the fact that not all individuals find the intrusions distressing could possibly explain the relatively low correlations between avoidance and the occurrence of intrusive memories at later stages of the disorder (see previous section). It is conceivable that the distress caused by intrusions rather than the frequency of their occurrence predicts avoidance, especially if assessments are taken long after the trauma. No published studies have investigated this hypothesis, and scales assessing severity of PTSD are measures of symptom presence/absence or frequency (Impact of Event Scale, IES, Horowitz et al., 1979; PTSD Symptom Scale, PSS, Foa, Riggs, Dancu and Rothbaum, 1993).

Preliminary data supporting the role of distress caused by intrusions are discussed below (Steil and Ehlers, in preparation). The variability in distress caused by intrusive recollections could be due to cognitive factors, i.e., 
different meanings of the intrusive memories for the individual (see suggestion 1).

The need to distinguish different phases of the disorder in establishing links between intrusions and persistent PTSD

An important finding of prospective studies of PTSD is that the experience of intrusive recollections immediately after the trauma is often not a good predictor of persistent PTSD (in terms of specificity) (McFarlane, 1988; Perry, Difede, Musngi, Frances and Jacobsberg, 1992; Shalev, 1992; but see the positive results for 3-month longitudinal studies of Rothbaum et al., 1992, and Winter and Ehlers, in preparation). McFarlane (1992b) mentioned that the presence of intrusive memories and avoidance in itself is not sufficient in differentiating adaptive trauma-related distress from the presence of PTSD "... the clinical significance of intrusive phenomena in the immediate aftermath of the trauma is different from that 3 to 4 months later" ( $p$. 599). Like Shalev (1992), McFarlane (1992b) assumed that arousal symptoms are crucial in predicting persistent PTSD.

However, this hypothesis does not explain why intrusions measured after several months have elapsed (4 months or later) were an impurtant predictor of persistent PTSD in the studies of MacFarlane (1992a), Perry et al. (1992) and Baum et al. (1993). The difference in pattern of results indicates that it is important to distinguish between factors determining the development of initial PTSD symptoms and those involved in their maintenance, as mentioned in the introduction.

\section{A cognitive approach to the maintenance of intrusive recollections}

In the following, we will discuss two suggestions for future research that are based on the considerations described above and the literature on other anxiety disorders (especially Salkovskis, 1985, in press) and depression (Teasdale and Barnard, 1993; Williams, Watts, MacLeod and Mathews, 1988). Preliminary results from our studies are presented. The suggestions are:

1. The idiosyncratic meaning of the intrusive recollections contributes to the persistence of the intrusions.

2. A comprehensive assessment of strategies used by the patient to control the occurrence of intrusive recollections is necessary to explain persistence of intrusive recollections. These strategies prevent change of negative idiosyncratic meanings of the trauma and of the intrusions. 
Suggestion 1: Role of negative idiosyncratic meaning of intrusions in persistent PTSD

We suggest that the patients' interpretations (i.e., the idiosyncratic meaning) of their intrusive recollections determine:

1. How distressing the intrusions are.

2. The extent to which the patient engages in strategies to control the intrusions.

3. These strategies prevent a change of meaning of the trauma and of the intrusive recollections. Thus, as a consequence of 2 , they contribute to the maintenance of intrusive recollections and PTSD symptoms in general (see suggestion 2 for possible mechanisms).

We define the content of negative interpretations of intrusive recollections as follows: The traumatized individual interprets intrusive memories as a sign of threat. In particular, the individual interprets the fact that the intrusive recollection has occurred and/or its particular content as indicating the possibility (but not certainty) of

- something negative about themselves which he/she finds unacceptable, or

- an ongoing threat that their world (i.e., their social relationships) presents to him/her, or

- a permanent damage that may have been done to him/herself (i.e., physical or mental health) or their world (i.e., their social relationships) which prevents him/her from going back to his/her normal life.

These negative interpretations lead to a sense of ongoing threat (anticipation of future negative events), and loss of a positive perspective for the future.

Note that we use the term interpretations in a broad sense. They include conscious appraisals that take the form of automatic thoughts as described by Beck (1976) and network associations with particular meanings as described by Lang (1977). What is important for our hypotheses is that the individual assigns a negative idiosyncratic meaning to his/her intrusive recollections that meets the criteria described above.

Examples of these negative interpretations are:

- A survivor of child sexual abuse interprets the intrusions as meaning she/ he is about to go crazy.

- A patient involved in a road traffic accident interprets the intrusions as a sign of a brain injury.

- Another accident patient interprets the intrusions as indicating that she/ he is going to suffer forever. 
- A rape patient interprets the intrusions as meaning she/he is inadequate because she/he cannot cope with stress.

- A patient experiencing intrusive memories during a conversation with a friend interprets these as indicating that she/he will never be able to form a close relationship with other people again.

The negative interpretation of intrusive recollections usually reflects maladaptive beliefs that the individual held before experiencing the trauma (e.g., beliefs about how long it is appropriate to be upset, beliefs about psychological symptoms). The interpretations may also be linked to a maladaptive belief formed as a result of the patient's interpretation of the trauma. In the latter case, the intrusive recollection may be interpreted as providing further confirmation of the interpretation of the trauma. For example, a patient who was injured due to the reckless driving of another driver developed the belief that the world is unjust. He interpreted the fact that he suffered from very distressing intrusions as yet another indication of this injustice.

We expect that patients for whom their intrusive memories have a negative meaning as described above experience more distress and are more likely to engage in strategies to control the intrusions (see suggestion 2) than patients who think the intrusions are a normal part of recovery. Since strategies employed to control the occurrence of intrusions prevent change in meaning, negative interpretations of intrusive memories should therefore contribute to the persistence of intrusions and other PTSD symptoms. It is also possible that maladaptive interpretations of intrusive recollections actually lead to changes in the traumatic memories themselves. Indirect evidence for the latter hypothesis comes from a study of Schwarz et al. (1993) who found that frequency of intrusions and other PTSD symptoms at initial screening were correlated with enlargement of diminishment of recall of sensory and emotional experiences (especially life threat) after a school shooting.

\section{Preliminary evidence for the role of interpretations of intrusive recollections} Wenninger and Ehlers (in preparation) conducted structured interviews of 35 adult survivors of child sexual abuse about their thoughts when experiencing recollections of the abuse. A subgroup of the women reported thoughts indicating negative interpretations, e.g., "I am bad", "I am stupid", "I am ugly", or "I am a failure, he was right". These women showed greater severity of posttraumatic symptoms and more dysfunctional cognitions in themes such as power, intimacy, self-respect, safety, and trust than women who did not report negative automatic thoughts.

In a questionnaire survey of 159 individuals involved in road traffic 
accidents, Steil and Ehlers (in preparation) found that the vast majority still experienced intrusive memories. Interestingly, $24 \%$ reported that they were not distressed by these intrusions. The nondistressed group did not differ from the distressed group in objective characteristics of the accident such as severity of injuries or time since the accident had occurred.

This study and another study of 138 individuals involved in road traffic accidents (Steil and Ehlers, in preparation) provided preliminary evidence for the role of negative interpretations of intrusive memories. Questionnaires assessing catastrophic interpretations differed between the studies. Study 1 was focused on the evaluation that intrusive memories meant that the subjects' mental health was in danger ("I am going crazy"). In study 2, a wider range of interpretations was assessed (e.g., "My life is ruined", "Something is seriously wrong with me", "I will never get over this", "I will not live long"). PTSD severity was assessed with the PTSD Symptom Scale (PSS, self-report version) developed by Foa et al. (1993). Cognitive and behavioural avoidance was assessed with a questionnaire specifically designed for road traffic accidents by Steil and Ehlers (in preparation).

The following hypotheses were derived from our suggestions on the role of interpretations of traumatic memories as outlined above:

1. The subjects' catastrophic interpretations of the intrusive traumatic memories explain a proportion of the variance of recollection-related distress which is not explained by intrusion frequency. Results: In both studies, highly significant partial correlations of $r=.42(p<.001)$ and $r=.48(p<$ .001) were found.

2. The subjects' catastrophic interpretation of the intrusive traumatic memories explains a proportion of the variance of how uncontrollable the intrusive memories appear which is not explained by intrusion frequency. Results: In study 1, a significant partial correlation of $r=.21(p=.01)$ was consistent with this hypothesis. In study 2 , two aspects of uncontrollability were assessed separately, both of which showed significant partial correlations with catastrophic interpretations, control over onset of recollections $(r=.41, p<.001)$ and ability to bring recollections to an end $(r=.53, p<$ $.001)$.

3. The subjects' catastrophic interpretation of the intrusive traumatic memories explains a proportion of the variance of behavioural and cognitive avoidance which is not explained by intrusion frequency. Results: In both studies partial correlations of $r=.46$ and $r=.42$ ( $p s<.001$ ) were consistent with this hypothesis.

4. PTSD severity is more closely related to distress caused by intrusive recollections than intrusion frequency. Results: In study 1, frequency of 
intrusive recollections showed a correlation with PTSD severity of $r=.39$ $(p<.001)$. A significant correlation is to be expected because intrusive recollections are one of the symptoms of PTSD and thus contribute to the overall score. A significantly higher correlation was observed for distress caused by intrusive recollections $(r=.63, p<.001$, difference $t=3.39, p<$ .001 , one-tailed). In study 2 , the same result was obtained ( $r=.44$ and $r=$ .59 , respectively, difference $t=1.82, p<.05$, one-tailed).

5. Catastrophic interpretations of traumatic memories are related to severity of PTSD. Results: In study 1, catastrophic interpretations of intrusive traumatic memories correlated with PTSD severity even if recollection frequency was controlled for (partial correlation: $r=.48, p<.001$ ). In study 2 , in which a wider range of catastrophic interpretations was assessed, the partial correlation was $r=.56(p<.001)$. In this study, catastrophic interpretations showed a stronger relationship to PTSD severity than frequency of intrusive recollections $(r=.61$ vs. $r=.44, t=2.10, p<.05$, onetailed).

All correlations between catastrophic interpretations and recollectioninduced distress, avoidance and PTSD severity remained significant when indicators of the severity of the accident (e.g., severity of injuries) and of the content of the intrusive recollections (e.g., number of dead or injured people seen by the patient) were partialled out.

These results support our suggestion that it is useful to distinguish between the occurrence of intrusive recollections, and the distress caused by them. Catastrophic interpretations seem to play a role in explaining recollection-related distress and PTSD-severity. We are currently conducting a prospective longitudinal study of intrusive memories in PTSD in cooperation with Dr Frommberger, University of Freiburg, Germany, to investigate the predictive validity of interpretations of intrusions in accident victims.

\section{Relationship to other models}

The idea that the interpretation of anxiety symptoms is a crucial factor in maintaining anxiety disorders builds on previous work on panic disorder (see Clark, 1986; Ehlers et al., 1988). Our particular suggestions for the role of interpretations of intrusive memories are inspired by Rachman's (Parkinson and Rachman, 1981; Rachman and de Silva, 1978) and Salkovskis's (1985) work on intrusive thoughts in patients with obsessive-compulsive disorder. Salkvoskis $(1985,1995)$ suggested that the misintepretation of intrusions (termed negative automatic thoughts about intrusions in the 1985 paper) is crucial in explaining maintenance of intrusions. Only if such 
minsinterpretations occur will the individual find the intrusion distressing: "The intrusions will only be expected to produce distress when they have some (idiosyncratic) meaning or salience to the individual experiencing them" (1985, p. 573), and "the discomfort experienced is due to the patient's appraisal of the content and occurrence of intrusive thought" (Salkovskis et al., 1995). The appraisal of intrusions in OCD centre on distorted beliefs about responsibility, i.e., the belief that one has power which is pivotal to bring about or prevent subjectively crucial negative outcomes (Salkovskis et al., 1995). According to Salkovskis (1985, Salkovskis et al., 1995) the idiosyncratic meaning of the intrusions determines the degree to which the individual engages in neutralization strategies, i.e. attempts to avoid or reduce the possibility of being responsible for harm to oneself or others, and in attempts at thought suppression. The neutralization strategies reduce distress, but maintain the disorder by preventing changes in the underlying dysfunctional beliefs about responsibility.

There are a number of important differences between intrusive memories in PTSD patients and intrusions in patients with obsessions. These have to be taken into account when applying Salkovskis's approach to a model of maintenance of intrusive memories in PTSD:

1. The intrusive memories in PTSD concern a catastrophe that did really happen whereas obsessive intrusions often concern hypothetical catastrophes that might happen in the future. The core interpretations in PTSD centre around the threat that this horrible event implies for the individual's future. In contrast, the core interpretations in OCD centre around the patient's responsibility for future catastrophes or, if they concern a past negative event, the patient's responsibility for minimizing the impact of this event (Salkovskis, personal communication).

2. Whereas distress and physiological arousal in OCD is solely attributed to automatic thoughts, the high physiological arousal that accompanies intrusive memories in PTSD is probably, at least initially, a direct part of the memory of the original event (see Foa et al.'s network model, 1989; Creamer et al., 1992) and thus automatically activated (i.e., not only a function of the distress caused by the interpretation). The high arousal may contribute to the negative interpretations of the intrusions discussed above, and may contribute to selective information processing (see suggestion 2) and thus make it more difficult to incorporate new corrective information.

3. The intrusive recollections occur predominantly in the form of visual images (see studies of Wenninger and Ehlers, in preparation, and Steil and Ehlers, in preparation, described above). This may make the idiosyncratic meaning appear more self-evident to the traumatized individual than if they 
were thoughts and therefore more difficult to change. The idiosyncratic meaning may also be experienced in the form of visual images.

Vaughan and Tarrier (1992) have also suggested that Salkovskis's model can be applied to PTSD. They described how PTSD patients often engage in a cognitive commentary when they experience intrusive memories. Examples of this cognitive commentary are a patient who thought about the unfairness of his employer's attitude towards him after the robbery, or a patient who ruminated about the murderer going free and nobody believing her. Vaughan and Tarrier made the important observation that these preoccupations terminate exposure (p. 663), in other words, they terminate the intrusive recollection. They also propose that this cognitive commentary is similar to the automatic thoughts (or misinterpretations of intrusions) described by Salkovskis for patients with OCD (p. 659).

However, the examples given in Vaughan and Tarrier (1992) do not represent examples of interpretations of intrusive memories although they probably reflect maladaptive beliefs. Instead, the cognitive commentary resembles the rumination observed in patients with GAD (see Borkovec, 1985; Mathews, 1990). Thus, they are likely to represent a form of cognitive avoidance (see suggestion 2) and are similar to the neutralization strategies described in Salkovskis's model. We propose that a clearer distinction of interpretations of intrusions and strategies used by the patient to control their occurrence has the following advantages: it enables researchers to investigate the functional role of interpretations in leading to strategies that prevent emotional processing and recovery, it explains how adaptive interpretations could facilitate recovery, and it makes it possible to conceptualize cognitive interventions designed to identify the idiosyncratic meaning of intrusions and modify the underlying assumptions directly.

Other authors have also alluded to the possible role of cognitive processing of PTSD symptoms in maintaining PTSD. For example, Horowitz (1976) pointed out that the distress caused by intrusions depends on the degree to which the recollections are perceived as controllable and that uncontrollable intrusions can lead to retraumatization. Jones and Barlow (1990) have developed a model of PTSD that assigns a central role to the development of anxious apprehension about "learned alarms", i.e., emotional responses that resemble the original "true alarm" response to the traumatic situation. Because learned alarms are experienced as unpredictable and uncontrollable they lead to a state of hyperarousal and hypervigilance for danger cues (see also Chemtob et al., 1988). Hyperarousal, like any stress, increases the frequency of intrusive thoughts and images, many of which are trauma-related. "The hypervigilance and attention narrowing on 
this threatening and dangerous material (trauma related intrusive thoughts), which might signal the onset of further uncontrollable emotional states, would also play an important role in the downward spiral of negative affect, paradoxically increasing, rather than decreasing, re-experiencing of trauma" (p. 319). Jones and Barlow (1990) describe a process of maintenance of trauma-related intrusive memories that involves repeated experiences of uncontrollable, negative emotional states and hyperarousal, and the anxious apprehension about repetitions of this experience which is accompanied by selective information processing. Thus, Jones and Barlow (1990) provide a framework for distinguishing between individuals who experience transient symptoms of PTSD and those who develop chronic PTSD.

Chemtob et al. (1988) proposed a positive feedback loop between thoughts and memories of threat (i.e., the trauma) and threat arousal that leads to selective attention to threat cues. This attentional bias may lead to the increased perception of reminders of the trauma, which in turn leads to more threat arousal and more intrusions. Chemtob et al. (1988) also mentioned that the perception of the physiological responses of threat arousal may provide the individual with further evidence of danger (see also Foa et al.'s model described below).

Jones and Barlow (1990) cite an interesting observation by McFarlane who found that many PTSD patients fail to associate their current symptoms with the traumatic experiences that ultimately led to the disorder. The lack of attribution to trauma could be involved in maintaining PTSD because symptoms are less predictable and cause more distress than if the cause was known to the patient (similar to panic attacks, see Goldstein and Chambless, 1978). Another aspect may be that if subjects do not have information on the source of their moods, they will evaluate their lives more negatively, as demonstrated by Schwarz and Clore (1983). In PTSD patients, unexplained symptoms of anxiety and depression may therefore contribute to the formation of negative beliefs about themselves or their world.

Foa et al. (1989) proposed that the meaning of fear response elements is a crucial component of fear networks and that patients with anxiety disorders assign negative meanings to fear responses ("badness", e.g., anxiety is impossible to tolerate, anxiety will never end). Foa and Riggs (1993) suggested that the presence of post-trauma symptoms is perceived by the rape victim as a sign of incompetence and her inability to cope. This perception in turn exacerbates PTSD symptoms.

Burstein (1989b) reported that experiencing psychological symptoms after a road traffic accident is often perceived as embarrassing. Individuals for whom anxiety and distress carry a high negative valence are more likely to engage in avoidance strategies. Foa et al. (1989) also proposed that exposure 
changes the threat meaning linked to response elements as well as stimulus elements. The patient experiences habituation during the sessions and the "badness" of high arousal can be decreased through repeated exposure.

In summary, other authors have also pointed to the role of negative meanings of PTSD symptoms in the maintenance of PTSD. A common assumption is that they lead the individual to engage in avoidance of reminders of the trauma and to selectively attend to potential signs of further threat. The focus in the work of most other authors has been on negative meaning of high arousal and unpleasant emotional states. We suggest that, in addition to looking at the implication of high arousal, the idiosyncratic meaning of having intrusive memories should be investigated.

\section{Suggestion 2: Bebavioural and cognitive processes used to control occurrence of intrusive recollections}

Since the pioneering work of Mowrer (1960), avoidance is the core construct used to explain maintenance of anxiety disorders. Situational or cognitive avoidance of reminders of the trauma are major symptoms of PTSD, but may not be sufficient in explaining maintenance. Several authors have pointed out that the concept of avoidance needs to be expanded in order to explain maintenance of PTSD and have given examples of the processes preventing recovery discussed in this section (e.g., Foa et al., 1989; Foa and Riggs, 1993; Horowitz, 1976; Spiegel, 1991).

In order to determine processes that maintain intrusive recollections, it is useful to consider mechanism of maintenance vs. recovery established for other anxiety disorders. The emotional processing concept (Rachman, 1990) provides a general framework for these considerations. Rachman (1990) defined emotional processing operationally as "a process where emotional disturbances are absorbed and decline to the extent that other behavior can proceed without disruption" (p. 282) and "a return to ongoing behavior after an emotional disturbance has waned" (p. 285), and gave examples for factors that impede emotional processing. These include avoidance, inadequate exposure or exposure under suboptimal conditions such as fatigue and persisting misinterpretations of threat.

What are the mechanisms of change in emotional processing? On the basis of the efficacy of exposure treatments, one of the core mechanisms proposed in the literature is habituation (see Foa and Kozak, 1986). Foa and Kozak (1986) have linked the process of habituation to a cognitive mechanism of change, i.e., the incorporation of new corrective information. Other authors have also emphasized the role of corrective information, but do not assume that fear arousal and habituation are necessary for achieving change (Rachman, 1990). Another important concept linked to emotional 
processing is the disconfirmation of expected negative outcomes (expectancy and avoidance: Seligman and Johnston, 1973; overprediction of fear and pain: Rachman, 1990). From a cognitive perspective, exposure can be interpreted as a method to induce belief change by guided hypothesis testing ("behavioral experiments", see, for example, Clark, 1989).

A common denominator of the proposed mechanisms leading to emotional processing is that they lead to a change in the maladaptive idiosyncratic meaning of anxiety-inducing stimuli. We therefore suggest maintenance of intrusive recollections can be explained by processes that prevent change in the idiosyncratic negative meaning of the trauma and of the intrusive recollections. In order to achieve a comprehensive assessment of these processes it may be useful to distinguish two ways by which change in meaning of traumatic memories may occur:

- New information changes the meaning the trauma or the intrusive memories have for the patient to the effect that the trauma is accepted by the patient as part of their experience and that the negative implications of the event and the recollections for the future are reduced.

- The organization of the memory structure is changed in a way that facilitates a change in meaning of the trauma or the intrusive memories. This change can concern the content of the memories (e.g., new important aspects of the trauma are remembered such as the rapist having a knife; the sequence of events is remembered such as the patient only complied with the rapist after he had threatened to kill her; the memory becomes less patchy) or the mode of representation of trauma in memory (e.g., transformation from a predominantly visual to a predominantly semantic representation).

There are several different pathways by which strategies used by the patient to control intrusive recollections interfere with a change of meaning of the trauma and the intrusive recollections (examples of which are discussed in the sections below): First, failure to expose oneself to the traumatic aspects of the event may lead to insufficient activation of the memory structure (Foa et al., 1989) for the meaning of its components and its organization to be changed (see section on rumination). Second, strategies used to control intrusive recollections may maintain maladaptive beliefs about the self, the world, and the meaning of symptoms (see section on safety behaviours). Third, attempts to control intrusive recollections may directly increase their frequency (see section on thought suppression). Failure of strategies to control the occurrence of intrusive recollections may be perceived as uncontrollable and may be interpreted by the patient as further evidence for maladaptive beliefs maintaining the disorder. Fourth, selective 
information processing may make it more likely that triggers of intrusive recollections are perceived, and that negative aspects of the traumatic memory and its sequelae are recalled and rehearsed.

On the basis of these considerations, we suggest considering the role of the following processes in maintaining intrusive recollections of traumatic events:

- behavioural processes: avoidance of reminders of trauma, not talking about trauma, safety behaviours, alcohol and drug (ab)use;

- cognitive processes: avoiding thinking about trauma, dissociation and numbing, suppression of memories and thoughts about trauma when they occur, rumination (worry), activation of other emotions (such as anger and guilt) and corresponding cognitions, attentional and memory biases.

In the following, we will outline evidence collected so far on the role of these processes in maintaining intrusive memories. If possible, we will include preliminary ideas on the possible mechanisms of maintenance.

Not talking about the trauma. Foa et al. (1989) mentioned that one common form of avoidance is refraining from talking about the trauma. DSM-IV (American Psychiatric Association, 1994) has included avoidance of conversations about the trauma among the typical examples of avoidance. Pennebaker (1989) conducted a series of studies showing that talking or writing about traumatic or very distressing events leads to decreases in psychological distress and has beneficial effects on subjects' health and immune function. Steil and Ehlers (in preparation) found that, in road traffic accident victims, reports of not being able to talk enough were related to P'TSD severity (study 1: $r=.36, p<.001$; study $2: r=.19, p<.05$ ). A 3-month prospective study supported this finding (Winter and Ehlers, in preparation).

The mechanisms by which talking or writing lead to emotional processing are not yet clear. According to Foa et al. (1989) one could assume that talking activates the fear network and habituation occurs which leads to a change in the fear structure. In addition, talking may help organizing fragmented memories (Foa and Riggs, 1993). However, contrary to the hypothesis that the activation of the fear structure has to be accompanied by arousal in order to be effective, Pennebaker (1989) found that disclosure was related to low skin conductance levels. Pennebaker (1989) assumed that not talking about a trauma is an effortful process (active inhibition) which leads to increased arousal and failure to fully process the event. During disclosure, active inhibition is given up which leads to a decrease in arousal. Pennebaker (1989) also mentioned that translating the distressing event 
into language may be an important mechanism of change: "Once encoded linguistically, individuals can more readily understand, find meaning in, or attain closure of the experience". It is possible that the different predictions derived from Foa et al.'s (1989) and Pennebaker's (1989) work are partly due to the samples studied. It is also possible that different processes of change operate. More data are needed on the relative contribution of physiological arousal and habituation and the verbal account of a trauma during exposure. The effectiveness of exposure treatment for PTSD developed by Foa and colleagues (Foa, Rothbaum, Riggs and Murdock, 1991) does not allow one to decide between these components because subjects imagine the rape and verbalize what they are imagining during exposure.

Safety behaviours. Kilpatrick and Resnick (1993) mentioned that they observed checking behaviour in crime victims similar to that observed in OCD. Any excessive precautions taken by the individual to prevent further trauma could be considered safety behaviours. Examples include not sleeping in order to be able to detect an intruder, or driving very slowly in order to prevent another accident. These safety behaviours prevent emotional processing because they maintain the conviction that the individual is not safe and that the trauma is likely to happen again. Patients believe that another trauma would have occurred if they had not engaged in the safety behaviour.

The concept of safety behaviours can also be directly applied to the maintenance of negative beliefs about the meaning of intrusive recollections. For example, a patient who interpreted his recollections as indicating he might have a brain injury frequently did memory exercises and mental arithmetic in order to improve his intellectual functioning. These exercises maintained his belief that his brain would not function well without special training.

Alcobol and drug (ab)use. As Schwartz (1990) pointed out, for some PTSD patients use of various substances is a way to self-medicate the symptoms. However, substance (ab)use prevents emotional processing and may even exacerbate the PTSD symptoms. In line with this hypothesis, Feinstein and Dolan (1991) found that subjects with high alcohol consumption were at greater risk of developing P'TSD after traumatic events involving injuries.

Dissociation. Dissociation during the trauma may be an adaptive response as described by Spiegel (1991): "The essence of trauma is physical helplessness.... When physical control is lost, mental control becomes 
paramount. One way to maintain control is to distance oneself from one's body" (p. 262). However, when used as a long-term strategy, dissociation becomes maladaptive and perpetuates psychological and social maladjustment (Spiegel, 1991, p. 265). Breslau and Davis (1992) found that detachment from others distinguished between individuals with chronic and nonchronic PTSD. Foa et al. (1989) proposed that cognitive strategies such as depersonalization, emotional numbness, distractibility, and memory loss prevent exposure to feared material and are not adaptive since they prevent emotional processing. Foa et al. (1992) mentioned that these dissociative symptoms are functionally similar to avoidance and escape behaviours since they provide an escape from distress, although they may represent separate factors according to recent results of Foa's group (Foa and Hearst-Ikeda, in press). According to Foa and Riggs (1993) dissociation is employed when active avoidance fails to reduce distress.

Spiegel (1991) elaborated on how dissociation could be involved in the maintenance of intense reliving experiences such as flashbacks. He describes that "There is an on-off quality to dissociative experiences" (p. 266). When the flashbacks occur, patients experience them as if they were happening at that moment and the subsequent history (what happened after the trauma) is dissociated. The tendency of patients to dissociate needs to be taken into account when treating PTSD patients. Foa and Hearst-Ikeda (in press) concluded that therapeutic techniques directly aimed at reducing dissociation are necessary in the treatment of PTSD patients who dissociated during exposure therapy. For a recent review of the role of dissociation in PTSD, see Foa and Hearst-Ikeda (in press).

Thought suppression. There is some, although not unequivocal, evidence that suppression of unwanted thoughts may increase their frequency (Wegner, 1989). Recent research shows that this applies to naturally occurring intrusive thoughts (Salkovskis and Campbell, 1994; Trinder and Salkovskis, 1994). It is likely that these results are applicable to the attempt to suppress the occurrence of trauma-related intrusive cognitions.

Rumination. PTSD patients frequently ruminate about issues like why the trauma happened to them or how things could have been different if they had done something differently. Analogous to worry in GAD (Mathews, 1990 ), this form of rumination may prevent emotional processing and could thus be involved in maintaining PTSD. Borkovec, Shadick and Hopkin's theory (1990) that the function of worry is to protect people from potentially distressing imagery and corresponding physiological arousal may apply to PTSD patients. Patients may engage in rumination in order to 
reduce intrusive images of the trauma and the corresponding arousal. This suggestion is similar to Vaughan and Tarrier's (1992) suggestion that a "cognitive commentary" prevents exposure to the CS, and in line with Wells's (Wells, 1994; Wells and Matthews, 1994) suggestion that worrying is a strategically driven conceptual activity for dealing with threat.

Because the rumination in P'TSD concerns a past event, it is also different from worry in GAD in several respects. In particular, it may be linked to feelings of guilt and anger which are related to poor outcome (see below). In addition, rumination may also change the memory structure because it leads to selective recall of memories and the tendency to attend to information consistent with feared outcomes (see Mathews, 1990). This suggestion is similar to Wells's proposal (Wells, 1994; Wells and Matthews, 1994) that, in addition to blocking effective emotional processing, worrying may lead to generalization of retrieval cues for threat-related material in memory, which increases the frequency of intrusions.

Consistent with a maintaining role of rumination, Steil and Ehlers (in preparation) found correlations between subjects' reports that they ruminated about a road traffic accident they had experienced and severity of PTSD symptoms (study 1: $r=.54, p<.001$; study $2: r=.45, p<.001$ ). A prospective study showed that rumination assessed shortly after the accident predicted PTSD severity three months later (Winter and Ehlers, in preparation). In an experimental study, Wells and Papageorgiou (submitted) found that verbal worry following exposure to a gruesome film increased frequency of intrusions in the three days following the experiment compared to a control condition (settling down).

The relationship of rumination with PTSD severity is especially interesting because rumination is not among the DSM-IV symptoms and thus is not a part of the assessment of PTSD severity. Future research will have to determine whether rumination is indeed a strategy employed by PTSD patients to avoid imagery and high arousal which prevents emotional processing or whether it is just a correlate of more negative interpretations of the event.

Other emotions and related cognitions. Foa and colleagues have demonstrated that guilt and anger predict the maintenance of PTSD symptoms (Riggs, Dancu, Gershuny, Greenberg and Foa, 1992; Riggs et al. 1991). Furthermore, Foa, Riggs, Massie and Yarczower (in press) have recently found that patients who reported more anger prior to treatment and displayed less fear during exposure therapy had a poor treatment outcome, whereas facial expressions of anxiety predicted good outcome.

The findings on anger are in line with studies showing that blaming 
others for threatening events is associated with impairments in emotional well-being and physical health (Tennen and Affleck, 1990). Riggs et al. (1992) explained the role of anger in maintaining PTSD in that anger interferes with the modification of the traumatic memory. They assumed two possible processes for this effect. First, an anger network is established which shares many of its stimulus elements with the fear network and thus is likely to be activated by similar stimuli. Activation of the anger network inhibits fear responses, and, according to Foa et al. (1989), inadequate activation of all elements of the fear network may prevent change. Second, in the absence of external stimuli, intrusive thoughts and images of the trauma activate fear that leads the individual to employ cognitive strategies such as the activation of anger in order to avoid feelings of anxiety.

An additional aspect may be that guilt and anger only arise after the traumatic event has occurred and if persistent may reflect problematic postevent elaboration. For instance, ruminations about what the individual could have done differently to prevent being raped and about her doubts whether she did not in any way encourage the rapist would lead to guilt, and at the same time prevent emotional processing of the fear and helplessness she experienced during the rape. A person involved in a traffic accident may ruminate about how unjust it is that the person causing the accident suffered fewer injuries than he/she did and may get very angry about this. The focus on the anger prevents the processing of traumatic aspects of the accident such as threat to life. In both examples, the guilt or anger and corresponding cognitions prevent the patient from accepting that the trauma has happened.

Alternatively, or additionally, anger and guilt may reflect problematic beliefs (Foa and Clark, 1994; Resick and Schicke, 1993) that maintain PTSD. The example of the accident patient indicates shattered assumptions about how just the world is. Beliefs linked to anger are generally likely to reflect themes of trust in others and power/control (Resick and Schicke, 1993) and fairness (Foa and Clark, 1994). Guilt is linked to thoughts that the patient has behaved or thought in a way that breaks his or her personal rules about what is right and fair (Foa and Clark, 1994) and general beliefs about selfworth (Resick and Schicke, 1993).

Attentional and memory biases. Possibly because of the classification of PTSD as an anxiety disorder, research on information processing has largely concentrated on attentional biases for trauma-related threat cues (e.g., Chemtob et al., 1988; Jones and Barlow, 1990; Litz and Keane, 1989. An attentional bias favouring the processing of trauma-related information was observed (e.g., Cassidy, McNally and Zeitlin, 1992; Foa, Feske, Murdock, Kozak and McCarthy, 1991; McNally, Kaspi, Riemann and Zeitlin, 1990). 
The attentional bias could increase the probability that cues triggering traumatic recollections are perceived, and could thus be involved in the maintenance of the intrusions.

Because traumatic memories concern a past event, and because several of the symptoms of PTSD resemble those of depressed patients (e.g., diminished interest in significant activities, restricted range of affect), more research is needed on memory biases. It is likely that some memory biases observed in depressed patients are applicable to PTSD, for example, selective recall of negative aspects of the patient's behaviour during the trauma, or of responses of other people after the traumatic event. These memory biases may also be involved in the maintenance of PTSD. In line with this hypothesis, McNally, Litz, Prassas, Shin and Weathers (1994) found a deficit in retrieving specific autobiographical memories in Vietnam veterans with PTSD similar to that observed in depressed patients.

\section{Possible implications for treatment}

If the correlational results from our research on individuals involved in road traffic accidents on the role of intepretations of intrusive memories are supported by further evidence from prospective studies, they underline the need to assess frequency of symptoms, their interpretation, and distress caused by them separately during the course of the disorder. Current scales assessing severity of P'TSD are measures of symptom presence/absence or frequency (IES, Horowitz et al., 1979; PSS, Foa et al., 1993). In our research, additional variance could be explained if separate frequency and distress ratings were obtained. Measures of interpretations of intrusive recollections and other PTSD symptoms may be important predictors of long-term outcome in PTSD.

If future studies confirm our hypothesis that interpretations of intrusive memories are involved in their maintenance, this has possible implications for treatment. Cognitive therapy challenging the patient's negative interpretation of their intrusive recollections directly may be a useful adjunct to exposure and cognitive restructuring of the idiosyncratic meaning of the trauma. Schwartz (1990) suggested treating intrusive recollections directly by using thought stopping and thought substitution procedures. However, these procedures have the disadvantages that they might reinforce the negative meaning the intrusive recollections have for the patient ("I am going crazy") and that failure to stop the recollections can represent an experience of uncontrollability. It is therefore preferable to challenge the meaning by socratic dialogue and behavioural experiments (see Clark, 1989). Foa and Clark (1994) give the example of a rape victim who experienced flashbacks when she tried to have sex with her boyfriend. She interpreted the occur- 
rence of the flashbacks as meaning that she no longer had any sexual feelings for her boyfriend. When this interpretation was challenged, she could see that she had misinterpreted the fact that the flashbacks stopped her feelings.

If the full range of strategies that could prevent change in idiosyncratic meaning of the trauma and of intrusive recollections are assessed in future studies of PTSD, this is likely to improve the prediction of persistent PTSD. The assessment and modification of the full range of strategies preventing change in meaning may also improve treatment outcome. For example, Vaughan and Tarrier (1992) describe that image habituation training, a form of exposure therapy, was not successful in some patients who dissociated or who focused on other emotions such as anger during the imagery. Modifications of these strategies may be necessary in order to achieve improvement (see Foa and Hearst-Ikeda, in press).

One might also speculate that the predominance of visual recollections might have implications for treatment in that direct restructuring of the visual images might be an important aspect of treatment.

\section{References}

American Psychiatric Association (1994). Diagnostic and Statistical Manual of Mental Disorders (4th Ed.) DSM-IV. Washington, DC: APA.

Baum, A., Cohen, L. and Hall, M. (1993). Control and intrusive memories as possible determinants of chronic stress. Psychosomatic Medicine 55, 274-286.

BECK, A.T. (1976). Cognitive Therapy and the Emotional Disorders. New York: International Universities Press.

BLANK, A.S. (1993). The longitudinal course of posttraumatic stress disorder. In J.R.T. Davidson and E.B. Foa (Eds). Posttraumatic Stress Disorder. DSM-IV and Beyond (pp. 3-22). Washington, DC: American Psychiatric Press.

Borkovec, T.D. (1985). The role of cognitive and somatic cues in anxiety and anxiety disorders: worry and relaxation-induced anxiety. In A.H. Tuma and J.D. Maser (Eds). Anxiety and the Anxiety Disorders (pp. 463-478). Hillsdale: Erlbaum.

Borkovec, T.D., Shadick, R. and Hopkins, M. (1990). The nature of normal and pathological worry. In R. Rapee and D.H. Barlow (Eds). Chronic Anxiety and Generalized Anxiety Disorder. New York: Plenum Press.

Breslau, N. and Davis, G.C. (1992). Posttraumatic stress disorder in an urban population of young adults: risk factors for chronicity. American Journal of Psychiatry 149, 671-675.

BRETT, E.A. and OSTROFF, R. (1985). Imagery and posttraumatic stress disorder: an overview. American Journal of Psychiatry 142, 417-424.

Burstein, A. (1989a). Intrusion and avoidance symptoms in PTSD. American Journal of Psychiatry 146, 15-18.

Burstein, A. (1989b). Posttraumatic stress disorder in victims of motor vehicle accidents. Hospital and Community Psychiatry 40, 295-297.

Calmoun, K.S. and Resick, P.A. (1993). Post-traumatic stress disorder. In D.H. 
Barlow (Ed). Clinical Handbook of Psychological Disorders (2nd ed) (pp. 48-98). New York: Guilford.

Cassidy, K.L., McNally, R.J. and Zeirtuin, S.B. (1992). Cognitive processing of trauma cues in rape victims with post-traumatic stress disorder. Cognitive Therapy and Research 16, 283-295.

Chemtob, C., Roitblat, H.l., Hamada, R.S., Carlson, J.G. and Twentyman, C.T. (1988). A cognitive action theory of post-traumatic stress disorder. Journal of Anxiety Disorders 2, 253-275.

Clark, D.M. (1986). A cognitive approach to panic. Behaviour Research and Therapy 24, 461-470.

ClakK, D.M. (1989). Anxiety states. In K. Hawton, P. Salkovskis, J. Kirk and D.M. Clark (Eds). Cognitive Behaviour Therapy for Psychiatric Problems (pp. 52-96). Oxford: Oxford University Press.

Creamer, M., Burgess, P. and Pattison, P. (1990). Cognitive processing in posttrauma reactions: some preliminary findings. Psychological Medicine 20, 597-604.

Creamer, M., Burgess, P. and Pattison, P. (1992). Reaction to trauma: a cognitive processing model. Journal of Abnormal Psychology 101, 452-459.

DAVEY, G.C.L. (1989). UCS revaluation and conditioning models of acquired fears. Behaviour Research and Therapy 27, 521-528.

Davidson, J.R.T. and FoA, E.B. (1993). Epilogue. In J.R.T. Davidson and E.B. Foa (Eds). Posttraumatic Stress Disorder. DSM-IV and Beyond (pp. 229-235). Washington, DC: American Psychiatric Press.

Eberly, R.E., Harkness, A.R. and Engdahl, B.E. (1991). An adaptational view of trauma response as illustrated by the prisoner of war experience. Journal of Traumatic Stress 4, 363-380.

Ehlers, A., Margraf, J. and Roth, W.T. (1988). Selective information processing, interoception, and panic attacks. In I. Hand and H.U. Wittchen (Eds). Panic and Pbobias 2 (pp. 129-148). Berlin: Springer.

Fairbank, J.A., Hansen, D.J. and FitTerling, J.M. (1991). Patterns of appraisal and coping across different stressor conditions among former prisoners of war with and without posttraumatic stress disorder. Journal of Consulting and Clinical Psychology 59, 274-281.

Feinstein, A. and Dolan, R. (1991). Predictors of post-traumatic stress disorder following physical trauma: an examination of the stressor criterion. Psychological Medicine 21, 85-91.

FlanNery, R.B. (1990). Social support and psychological trauma: a methodological review. Journal of Traumatic Stress 3, 593-611.

FoA, E.B. and Clark, D.M. (1994). Therapist guide for cognitive restructuring. Unpublished manuscript.

Foa, E.B., Feske, U., Murdock, T.B., Kozak, M.J. and McCarthy, P.R. (1991). Processing of threat-related information in rape victims. Journal of Abnormal Psychology 100, 156-162.

FOA, E.B. and HEARST-IKEDA, D. (in press). Emotional dissociation in response to trauma: an information processing approach. In L.K. Michelson and W.J. Ray 
(Eds). Handbook of Dissociation: Theoretical, Einpirical, and Research Perspectives. New York: Plenum Press.

FoA, E.B. and KozAK, M.J. (1986). Emotional processing of fear: exposure to corrective information. Psychological Bulletin 99, 20-35.

FOA, E.B. and Riggs, D.S. (1993). Post-traumatic stress disorder in rape victims. American Psychiatric Press Review of Psychiatry 12 Washington DC: Author.

FoA, E.B., Riggs, D.S., Dancu, C.V. and Rothbaum, B.O. (1993). Reliability and validity of a brief instrument for assessing post-traumatic stress disorder. Journal of Traumatic Stress 6, 459-473.

FoA, E.B., Riggs, D.S., Massie, E.D. and Yarczower, M. (in press). The impact of fear activation and anger on the efficacy of exposure treatment for PTSD. Behavior Therapy.

Foa, E.B., Rothbaum, B.O., Riggs, D.S. and Murdock, T.B. (1991). Treatment of posttraumatic stress disorder in rape victims: a comparison between cognitivebehavioral procedures and counselling. Journal of Consulting and Clinical Psychology 59, 715-723.

FoA, E.B., Steketee, G. and Rothbaum, B.O. (1989). Behavioral/cognitive conceptualizations of post-traumatic stress disorder. Behavior Therapy 20, 155-176.

FoA, E.B., ZinbarG, R. and ROTHBAUM, B.O. (1992). Uncontrollability and unpredictability in post-traumatic stress disorder: an animal model. Psychological Bulletin 112, 218-238.

Freedy, J.R., Shaw, D.L., Jarrell, M.P. and Masters, C.R. (1992). Towards an understanding of the psychological impact of natural disasters: an application of the conservation resources stress model. Journal of Traumatic Stress 5, 441-454.

GLOVER, H. (1992). Emotional numbing: a possible endorphine-mediated phenomenon associated with post-traumatic stress disorders and other allied psychopathologic states. Journal of Traumatic Stress 5, 643-675.

Goldstein, A.J. and Chambless, D.L. (1978). A reanalysis of agoraphobia. Bebavior Therapy 9, 47-59.

GreEN, B.L. (1993). Disasters and posttraumatic stress disorder. In J.R.T. Davidson and E.B. Foa (Eds). Posttraumatic Stress Disorder. DSM-IV and Beyond (pp. 75-97). Washington, DC: American Psychiatric Press.

Horowitz, M.J. (1976). Stress Response Syndromes. New York: Aronson.

Horowitz, M., Wilner, N. and Alvarez, W. (1979). Impact of Event Scale: a measure of subjective stress. Psychosomatic Medicine 41, 209-218.

JanOFF-Bulman, R. (1985). The aftermath of victimization: rebuilding shattered assumptions. In C. R. Figley (Ed). Trauma and its Wake: The Study and Treatment of Posttraumatic Stress Disorder (pp. 15-35). New York: Brunner \& Mazel.

Janoff-Bulman, R. and Frieze, I.H. (1983). A theoretical perspective for understanding reactions to victimization. Journal of Social Issues 37, 105-122.

JONES, J.C. and BARLOW, D.H. (1990). The etiology of posttraumatic stress disorder. Clinical Psychology Review 10, 299-328.

Joseph, S., Yule, W. and Williams, R. (1993). Post-traumatic stress: attributional aspects. Journal of Traumatic Stress 6, 501-513. 
KilPatrick, D.G. and Resnick, H.S. (1993). Posttraumatic stress disorder associated with exposure to criminal victimization in clinical and community populations. In J.R.T. Davidson and E.B. Foa (Eds). Posttraumatic Stress Disorder. DSM-IV and Beyond (pp. 113-143). Washington, DC: American Psychiatric Press.

Kilpatrick, D.G., Saunders, B.E., Amick-McMullan, A., Best, C.L., Veronen, L. and Resick, H. (1989). Victim and crime factors associated with the development of post-traumatic stress disorder. Behavior Therapy 20, 199-214.

LANG, P.M. (1977). Imagery in therapy: an information processing analysis of fear. Behavior Therapy 8, 862-886.

LITZ, B.T. and KEANE, T.M. (1989). Information processing in anxiety disorders: application to the understanding of posttraumatic stress disorder. Clinical Psychology Review 9, 243-257.

Mathews, M. (1990). Why worry? The cognitive function of anxiety. Behavior Research and Therapy 28, 455-468.

Mayou, R., Bryant, B. and Duthie, R. (1993). Psychiatric consequences of road traffic accidents. British Medical Journal. 307, 647-651.

McCann, I.L. and Pearlman, L.A. (1990). Psychological Trauma and the Adult Survivor: Theory, Therapy, and Transformation. New York: Brunner and Mazel.

McFariane, A.C. (1988). The phenomenology of posttraumatic stress disorders following a natural disaster. Journal of Nervous and Mental Disease 176, 22-29.

McFarLane, A.C. (1992a). Avoidance and intrusion in posttraumatic stress disorder. Journal of Nervous and Mental Disease 180, 439-445.

McFarlane, A.C. (1992b). Commentary. Posttraumatic stress disorder among injured survivors of a terrorist attack: predictive value of early intrusion and avoidance symptoms. The Journal of Nervous and Mental Disease 180, 599-560.

McNally, R.J., Kaspi, S.P., Riemann, B.C. and Zeitlin, S.B. (1990). Selective processing of threat cues in posttraumatic stress disorder. Journal of Abnormal Psychology 99, 398-402.

McNally, R.J., Litz, B.T., Prassas, A., Shin, L.M. and Weathers, F.W. (1994). Emotional priming of autobiographical memory in post-traumatic stress disorder. Cognition and Emotion 8, 351-367.

Mowrer, O.H. (1960). Learning Theory and Behavior. New York: Wiley.

Parkinson, L. and Rachman, S. (1981). Part II. The nature of intrusive thoughts. Advances in Behaviour Research and Therapy 3, 101-110.

Pennebaker, J.W. (1989). Confession, inhibition, and disease. Advances in Experimental Social Psychology 22, 211-244.

Perry, S., Difede, J., Musngi, G., Frances. A.J. and Jacobsberg, L. (1992). Predictors of posttraumatic stress disorder after burn injury. American Journal of Psychiatry 149, 931-935.

Rachman, S.J. (1990). Fear and Courage (2nd ed.). New York: Freeman.

Rachman, S.J. and DE Silva, P. (1978). Abnormal and normal obsessions. Behaviour Research and Therapy 16, 101-110.

Resick, P.A. and SchICKE, M.K. (1993). Cognitive Processing Therapy for Rape Victims. Newbury Park, California: Sage. 
Riggs, D., Foa, E.B., Rothbaum, B.O. and Murdock, T. (1991). Post-traumatic stress disorder following rape and non-sexual assault: a predictive model. Unpublished manuscript.

Riggs, D.S., Dancu, C.V., Gershuny, B.S., Greenderg, D. and Foa, E.B. (1992). Anger and post-traumatic stress disorder in female crime victims. Journal of Traumatic Stress 5, 613-625.

RothBaum, B.O. and FOA, E.B. (1993). Subtypes of posttraumatic stress disorder and duration of symptoms. In J.R.T. Davidson and E.B. Foa (Eds). Posttraumatic Stress Disorder. DSM-IV and Beyond (pp. 23-35). Washington, DC: American Psychiatric Press.

Rothbaum, B.O., Foa, E.B., Riggs, D.S., Murdock, T. and Walsh, W. (1992). A prospective examination of post-traumatic stress disorder in rape victims. Journal of Traumatic Stress 5, 455-475.

SAlkovskis, P.M. (1985). Obsessional-compulsive problems: a cognitive-behavioural analysis. Behaviour Research and Therapy 23, 571-583.

Salkovskis, P.M., Richards, H.C. and Forrester, E. (1995). The relationship between obsessional problems and intrusive thoughts. Behavioural and Cognitive Psychotherapy 23, 281-299.

SAlkovskis, P.M. and CAmpbell, P. (1994). Thought suppression induces intrusion in naturally occurring negative thoughts. Behaviour Research and Therapy 32, 1-8.

SCHWARTZ, L.S. (1990). A biopsychosocial treatment approach to post-traumatic stress disorder. Journal of Traumatic Stress 3, 221-238.

Schwarz, N. and Clore, G.L. (1983). Mood, misattribution, and judgements of well-being: informative and directive functions of affective states. Journal of Personality and Social Psychology 45, 513-523.

SCHwarz, E.D., Kowalski, J.M. and McNally, R.J. (1993). Malignant memories: post-traumatic changes in memory in adults after a school shooting. Journal of Traumatic Stress 6, 545-553.

Seligman, M.E.P. and Johnston, J.C. (1973). A cognitive theory of avoidance learning. In F.J. McGuigan and D.B. Lumsden (Eds). Contemporary Approaches to Conditioning and Learning. New York: Wiley.

Shalev, A.Y. (1992). Posttraumatic stress disorder among injured survivors of a terrorist attack. Predictive value of early intrusion and avoidance symptoms. Journal of Nervous and Mental Disease 180, 505-509.

Shalev, A.Y., Schreiber, S. and Galai, T. (1993). Early psychological responses to traumatic injury. Joumal of Traumatic Stress 6, 441-450.

Solomon, Z., Mikulincer, M. and Avitzur, E. (1988). Coping, locus of control, social support, and combat-related posttraumatic stress disorder: a prospective study. Journal of Personality and Social Psychology 55, 279-285.

Solomon, Z., Mikulincer, M. and Flum, H. (1988). Negative life events, coping responses, and combat-related psychopathology: a prospective study. Journal of Abnormal Psychology 97, 302-307.

SPIEGEL, D. (1991). Dissociation and trauma. In A. Tasman and S.M. Goldfinger 
(Eds). Review of Psychiatry (Vol. 10, pp. 261-275). Washington DC: American Psychiatric Press.

Steil, $R$. and Ehlers, A. (in preparation). Cognitive correlates of intrusive memories after road traffic accidents.

Teasdale, J.D. and Barnard, P.J. (1993). Affect, Cognition, and Change. Hillsdale, NJ: Lawrence Erlbaum.

Tennen, H. and AfFleck, G. (1990). Blaming others for threatening events. Psychological Bulletin 108, 209-232.

Trinder, H. and Salkovskis, P.M. (1994). Personally relevant intrusions outside the laboratory: long-term suppression increases intrusion. Bebaviour Research and Therapy 32, 833-842.

Van Der Kolk, B.A. and Saporta, J. (1991). The biological response to psychic trauma: mechanisms and treatment of intrusion and numbing. Anxiety Research 4, 199-212.

VAUghaN, K. and TARRIER, N. (1992). The use of image habituation training with posttraumatic stress disorder. British Journal of Psychiatry 161, 658-664.

Wegner, D.M. (1989). White Bears and Other Unwanted Thoughts: Suppression, Obsession, and the Psychology of Mental Control. New York: Viking.

Wells, A. (1994). Attention and the control of worry. In G.C.L. Davey and F. Tallis (Eds). Worrying: Pespectives on Theory, Assessment, and Treatment (pp. 91-114). New York: Wiley.

Wells, A. and Matthews, G. (1994). Attention and Emotion: A Clinical Perspective. Hove, UK: Lawrence Erlbaum.

Wells, A. and Papageorgiou, C. (submitted). Worry and the incubation of intrusive images following stress.

WenNinger, K. and EHLers, A. (submitted). Dysfunctional cognitions and adult functioning in child sexual abuse survivors.

WeNNINGER, K. and EHLERs, A. (in preparation). Nature of intrusive recollections in child sexual abuse survivors.

Williams, J.M.G., Watts, F.N., MacLeod, C. and Mathews, A. (1988). Cognitive Psychology and Emotional Disorders. Chichester: Wiley.

WINTER, $\mathrm{H}$. and EhLERs, A. (in preparation). Predictors of posttraumatic stress disorder after road traffic accidents. 